\title{
Fatores associados ao tempo da morte de vítimas de trauma: estudo de coorte retrospectivo*
}

Factors associated to the time of death of trauma victims: A retrospective cohort study

Factores asociados al momento de la muerte de víctimas de traumatismos: estudio de cohorte retrospectivo

\section{Daniela Vieira de Andrade Batista ${ }^{\mathrm{I}}$, Carolina Cassiano ${ }^{\mathrm{II}}$, Luciana Maria Capurro de Queiroz ObergIII, Daniele Muñoz Gianvecchio ${ }^{\mathrm{IV}}$, Regina Marcia Cardoso de Sousav ${ }^{\mathrm{v}}$, Lilia de Souza Nogueira ${ }^{\mathrm{VI}}$}

\begin{abstract}
Resumo: Objetivo: identificar os fatores associados ao tempo da morte de vítimas de trauma. Método: coorte retrospectiva que analisou laudos de autópsia de vítimas de trauma admitidas em 2015 no Instituto Médico Legal Central de São Paulo, Brasil. O tempo da morte foi identificado a partir do momento da ocorrência do trauma até a confirmação do óbito. O modelo linear generalizado foi aplicado para análise dos dados. Resultados: entre as 1.500 vítimas fatais (75,7\% homens; idade média 49,7 anos), prevaleceram as quedas (33,5\%), seguidas das agressões (27,8\%). Os fatores associados ao tempo da morte foram número de regiões corporais afetadas $(\mathrm{p}<0,001)$, tipo de trauma $(\mathrm{p}<0,001)$, sexo $(\mathrm{p}=0,009)$, gravidade do trauma segundo New Injury Severity Score $(\mathrm{p}<0,001)$, agressões $(\mathrm{p}<0,001)$ e lesões autoprovocadas intencionalmente $(\mathrm{p}<0,001)$. Conclusão: mulheres, vítimas de traumas que envolveram intencionalidade de provocar a morte e com elevada gravidade apresentaram tempo de sobrevida reduzido após a(s) lesão(ões).
\end{abstract}

Descritores: Ferimentos e Lesões; Índices de Gravidade do Trauma; Autopsia; Fatores de Risco; Mortalidade

\footnotetext{
${ }^{\text {I }}$ Enfermeira, mestre em ciências, doutoranda pelo Programa de Pós-Graduação em Enfermagem na Saúde do Adulto da Escola de Enfermagem da Universidade de São Paulo, São Paulo, SP, Brasil. E-mail: silva.danivieira2@gmail.com. Orcid: https://orcid.org/0000-0003-1035-7620.

II Enfermeira, especialista em gestão hospitalar e terapia intensiva, mestranda pelo Programa de Pós-Graduação em Enfermagem na Saúde do Adulto da Escola de Enfermagem da Universidade de São Paulo, São Paulo, SP, Brasil. E-mail: carolina.cassiano@usp.br. Orcid: https://orcid.org/0000-0003-0681-1827.

III Enfermeira, mestre em enfermagem, doutoranda pelo Programa de Pós-Graduação em Enfermagem na Saúde do Adulto da Escola de Enfermagem da Universidade de São Paulo, São Paulo, SP, Brasil. E-mail: luciana.oberg@globo.com. Orcid: https://orcid.org/0000-0002-1443-7081.

IV Médica, especialista em medicina legal e perícias médicas, Seção Técnica da Equipe de Perícias Médico-Legais Bem Me Quer do Instituto Médico Legal, São Paulo, SP, Brasil. E-mail: danimunoz@uol.com.br. Orcid: https://orcid.org/0000-0001-6188-6130.

V Enfermeira, doutora em enfermagem, docente do Departamento de Enfermagem Médico-Cirúrgica da Escola de Enfermagem da Universidade de São Paulo, São Paulo, SP, Brasil. E-mail: vian@usp.br. Orcid: https://orcid.org/0000-0002-2575-7937.

VI Enfermeira, doutora em ciências, docente do Departamento de Enfermagem Médico-Cirúrgica da Escola de Enfermagem da Universidade de São Paulo, São Paulo, SP, Brasil. E-mail: lilianogueira@usp.br. Orcid: http://orcid.org/0000-0001-5387-3807.

* Extraído da dissertação "Distribuição temporal das mortes de vítimas de trauma e fatores associados”, Programa de Pós-Graduação em Enfermagem na Saúde do Adulto (PROESA), Escola de Enfermagem da Universidade de São Paulo, 2017.
} 
Fatores associados ao tempo da morte de vítimas de trauma: estudo de coorte retrospectivo |2

\begin{abstract}
Objective: to identify factors associated to the time of death of trauma victims. Method: a retrospective cohort that analyzed autopsy reports of trauma victims admitted in 2015 at the Central Institute of Forensic Medicine of São Paulo, Brazil. The time of death was identified from the trauma moment to the confirmation of death. The generalized linear model was applied to analyze the data. Results: among the 1,500 fatal victims $(75.7 \%$ were men; mean age of 49.7 years old), falls prevailed (33.5\%), followed by aggressions (27.8\%). The factors associated with the time of death were the number of body areas affected $(\mathrm{p}<0.001)$, type of trauma $(\mathrm{p}<0.001)$, gender $(\mathrm{p}=0.009)$, trauma severity according to the New Injury Severity Score $(\mathrm{p}<0.001)$, assaults $(\mathrm{p}>0.001)$, and intentionally self-harm injuries $(\mathrm{p}<0.001)$. Conclusion: women, victims of traumas with the intention of causing death and with high severity presented a reduced survival time after the injury(ies).
\end{abstract}

Descriptors: Wounds and Injuries; Trauma Severity Indices; Autopsy; Risk Factors; Mortality

Resumen: Objetivo: identificar los factores asociados al momento de la muerte de víctimas de traumatismos. Método: estudio de cohorte retrospectivo que analizó informes de autopsias de víctimas de traumatismos admitidas en 2015 en el Instituto Médico Legal Central de San Pablo, Brasil. El momento de la muerte se identificó a partir del momento en que sucedió el traumatismo hasta la confirmación de la muerte. Se aplicó el modelo lineal generalizado para el análisis de los datos. Resultados: entre las 1.500 víctimas fatales $(75,7 \%$ de ellas hombres; media de edad de 49,7 años), prevalecieron las caídas (33,5\%), seguidas por las agresiones (27,8\%). Los factores asociados al momento de la muerte fueron la cantidad de áreas del cuerpo afectadas $(\mathrm{p}<0,001)$, el tipo de traumatismo $(\mathrm{p}<0,001)$, el sexo ( $\mathrm{p}=0,009)$, la gravedad del traumatismo conforme al New Injury Severity Score $(\mathrm{p}<0,001)$, agresiones $(\mathrm{p}<0,001)$ y lesiones autoprovocadas intencionalmente $(\mathrm{p}<0,001)$. Conclusión: las mujeres, víctimas de traumatismos con intención de provocar la muerte y de alta gravedad presentaron un tiempo de sobrevida reducido después de la o las lesiones.

Descriptores: Heridas y Traumatismos; Índices de Gravedad del Trauma; Autopsia; Factores de Riesgo; Mortalidad

\title{
Introdução
}

O trauma é um complexo e crescente problema de saúde pública que atinge a sociedade mundial. ${ }^{1}$ No Brasil, em 2018, a totalidade dos acidentes e violências representou a quarta principal causa de óbito, precedida por doenças cardiovasculares, neoplasias e doenças respiratórias. ${ }^{2}$ Ainda nesse ano, os eventos traumáticos vitimaram, na maioria, indivíduos entre 20 e 49 anos, ${ }^{2}$ população economicamente ativa, resultando, assim, em elevado número de anos potenciais de vida perdidos. Nesse contexto, desenvolver estudos que ajudem a minimizar o impacto do trauma na saúde e mortalidade da população tem sido um desafio para pesquisadores de diversos países.

Ao analisar as mortes relacionadas ao trauma, foi proposta a distribuição trimodal dos óbitos, dividindo a ocorrência de morte das vítimas de trauma em três períodos ou picos 
3 | Batista DVA, Cassiano C, Oberg LMC, Gianvecchio DM, Sousa RMC, Nogueira LS

específicos: imediato (mortes que ocorrem minutos após a lesão), precoce (mortes que acontecem entre a primeira e a quarta hora após a lesão) e tardio (mortes que ocorrem dias ou semanas após o trauma). ${ }^{3}$ Desde a sua publicação, a distribuição trimodal clássica dos óbitos passou a ser referência para pesquisadores que investigam o tema.

Recentes pesquisas identificaram diferentes períodos da distribuição temporal dos eventos fatais por trauma. ${ }^{4-10} \mathrm{O}$ período das mortes imediatas foi confirmado e está bem definido na literatura, ou seja, pico de óbitos no local do trauma ${ }^{5}$ ou dentro da primeira hora pós-lesão..$^{6-8}$ Entretanto, em relação ao pico precoce, o período identificado nas pesquisas varia de menos de uma hora a 48 horas ou mais, sendo que, para o tardio, esse tempo oscila de quatro horas a mais de sete dias. ${ }^{9-10}$

Além disso, pesquisadores retrataram mudança na distribuição das mortes por trauma, passando de trimodal para bimodal (pico tardio não foi observado), ${ }^{4,8,11}$ unimodal (um único pico acentuado na cena do trauma) ${ }^{5}$ ou em contínuo declínio ao longo do tempo. ${ }^{12}$ Tal mudança foi associada, principalmente, a melhorias no atendimento inicial e tratamento precoce e definitivo das vítimas, especialmente na "Golden Hour" (primeira hora após a lesão), considerada período crucial e determinante das chances de sobrevida das vítimas gravemente feridas. ${ }^{13}$ Dessa forma, é essencial que o tempo resposta, definido como tempo entre a notificação da ocorrência e a chegada da equipe pré-hospitalar na cena, seja o menor possível, para que as condutas que determinam a redução das mortes imediatas sejam realizadas e o encaminhamento rápido das vítimas para serviços estruturados para o cuidado definitivo esteja assegurado. ${ }^{14}$

Pesquisadores que analisaram o tempo de sobrevida (intervalo de tempo entre a hora do trauma e a declaração do óbito) de vítimas de trauma por acidentes de trânsito e homicídios identificaram que a maioria dos óbitos ocorreu nos primeiros minutos após o evento. Notaram também um segundo pico (35 a 40 minutos) entre as vítimas atendidas no hospital, que consideraram um possível resultado do adiamento da declaração do óbito em razão das tentativas de reanimação cardiopulmonar dos traumatizados. ${ }^{15}$ Estudo de revisão concluiu que a 
Fatores associados ao tempo da morte de vítimas de trauma: estudo de coorte retrospectivo 4

variação nos intervalos temporais utilizados para analisar a distribuição das mortes por trauma é tão ampla que se torna difícil identificar qual distribuição é a mais assertiva. ${ }^{16}$

Recentemente, investigadores avançaram na análise das mortes por trauma e pesquisaram as variáveis preditoras desse desfecho nos diferentes períodos identificados a partir da distribuição temporal. No entanto, como descrito anteriormente, a diversidade do tempo da morte considerado na distribuição temporal levou a diferentes descobertas sobre os fatores de risco nas casuísticas investigadas, sendo que as seguintes variáveis preditoras se destacaram: traumatismo cranioencefálico, lesões no tórax e abdome, idade avançada e choque hemorrágico. ${ }^{6,8,10}$ Analisando os estudos ${ }^{6,8,10}$ sobre distribuição temporal das mortes por trauma e os respectivos fatores preditores, observou-se, além de incongruências nos resultados, ausência de pesquisas que buscaram identificar os fatores associados ao tempo da morte de vítimas de trauma considerando o período do trauma até o óbito como variável contínua.

Portanto, a presente investigação tem a intenção de contribuir para atenuar essa lacuna do conhecimento e responder à seguinte questão de pesquisa: quais são os fatores associados ao tempo da morte das vítimas de trauma? A identificação de grupos de vítimas de trauma com características que determinam o tempo até sua morte é essencial para a criação e o aperfeiçoamento de políticas públicas de prevenção e assistência ao trauma, estratégias de capacitação das equipes que prestam atendimento às vítimas de trauma e otimização dos recursos humanos e materiais, com foco na prestação de cuidados de qualidade e redução de custos e de eventos fatais. Sendo assim, o objetivo deste estudo foi identificar os fatores associados ao tempo da morte das vítimas de trauma.

\section{Método}

Trata-se de uma pesquisa de coorte retrospectiva que analisou laudos de autópsia de vítimas de trauma admitidas no Instituto Médico Legal (IML) Central de São Paulo, Brasil. Esse 
5 | Batista DVA, Cassiano C, Oberg LMC, Gianvecchio DM, Sousa RMC, Nogueira LS

instituto é subordinado à Superintendência da Polícia Técnico-Científica e fornece bases técnicas em Medicina Legal para auxiliar no julgamento de causas criminais. O IML Central tem cobertura dos casos de óbitos ocorridos nas regiões oeste e centro da cidade de São Paulo.

A amostra foi composta por vítimas fatais de trauma admitidas no IML Central em 2015. Foram excluídos os indivíduos que não tiveram a hora da morte identificada e os que apresentaram descrições de lesões traumáticas nos laudos de autópsia de forma não codificável pela Abbreviated Injury Scale (AIS). Esta é uma escala usada para descrever lesões resultantes do trauma e identificar sua gravidade, cujo escore pode variar de 1 (lesão leve) a 6 (lesão de gravidade máxima, incompatível com a vida). ${ }^{17}$ Casos de afogamento ou envenenamento foram excluídos, pois a fisiopatologia dessas condições difere dos traumas contusos e penetrantes incluídos na investigação.

A variável dependente da pesquisa foi o tempo da morte, ou seja, tempo entre a ocorrência do evento traumático e a confirmação do óbito. As variáveis independentes analisadas foram idade, sexo, raça, tipo de trauma (contuso ou penetrante), causa externa de morbimortalidade de acordo com o capítulo XX da Classificação Estatística Internacional de Doenças e Problemas Relacionados à Saúde - 10ª Revisão (CID-10), ${ }^{18}$ gravidade do trauma segundo o Injury Severity Score (ISS) ${ }^{17}$ e o New Injury Severity Score (NISS), ${ }^{19}$ número de regiões corpóreas afetadas, região do corpo mais gravemente ferida e causa da morte descrita pelo médico legista.

O ISS é um índice anatômico de gravidade identificado a partir do escore AIS. Para calcular o ISS, são consideradas seis regiões do corpo (cabeça/pescoço, face, tórax, abdome/conteúdo pélvico, extremidades/cintura pélvica e superfície externa), sendo o escore do índice obtido pela soma dos quadrados dos escores AIS mais altos de três regiões diferentes do corpo. ${ }^{17}$ A principal crítica ao ISS está relacionada ao fato de que o índice não considera, para o cálculo, mais de uma lesão ocorrida em uma mesma região corpórea, podendo subestimar a gravidade do trauma. Para corrigir tal limitação, foi criado o NISS, calculado a partir da soma dos quadrados dos 
Fatores associados ao tempo da morte de vítimas de trauma: estudo de coorte retrospectivo 6

escores AIS mais altos, independentemente da região corpórea acometida. ${ }^{19}$ A pontuação do ISS e do NISS varia de 1 a 75 pontos, sendo que escores $<16$ caracterizam trauma leve, $\geq 16$ e $<25$ trauma moderado e $\geq 25$, trauma grave. ${ }^{17,19}$ Para o cálculo dos dois índices, é essencial identificar todas as lesões traumáticas da vítima.

A coleta de dados ocorreu entre março e setembro de 2016, com a leitura dos laudos de autópsias, transcrição das lesões traumáticas para cálculo do ISS e NISS e, sempre que necessário, a complementação da identificação do tempo da morte foi realizada por meio do acesso aos boletins de ocorrência das vítimas. Para a realização das análises descritivas e inferenciais do estudo, foram utilizados os programas Statistical Package for the Social Science - versão $22\left(\right.$ SPSS-22 ${ }^{\circledR}$ ) e R 3.3.2 ${ }^{\circledR}$. Um modelo linear generalizado para distribuição gama e função de ligação log foi desenvolvido para identificar os fatores associados ao tempo da morte das vítimas de trauma. O Fator de Inflação de Variância (VIF) foi aplicado para detectar a probabilidade de colinearidade múltipla entre as variáveis que permaneceram no modelo final, sendo que valores de VIF $\geq 5$ foram indicativos de colinearidade. O teste pseudo- $\mathrm{r}^{2}$ de Nagelkerke foi utilizado para avaliar a capacidade preditiva do modelo. O nível de significância adotado nas análises foi de $5 \%$.

A presente pesquisa foi conduzida de acordo com os padrões éticos exigidos, definidos pela Resolução 466/2012 do Ministério da Saúde, e foi aprovada pelo Comitê de Ética em Pesquisa da instituição, em 11/12/2015 (parecer número 1.363.957). Por se tratar de análise de vítimas fatais, foi autorizada, por esse comitê, a dispensa da aplicação do termo de consentimento livre e esclarecido aos familiares.

\section{Resultados}

Durante o período estudado, 1.679 admissões no IML Central foram por causas traumáticas fatais. Entre essas vítimas, 179 não atenderam aos critérios de inclusão do estudo, sendo que em 54 
casos não foi possível identificar o tempo da morte, 116 eram vítimas de afogamento ou envenenamento e 9 não tinham lesões traumáticas codificáveis pela AIS. Portanto, participaram da análise desta pesquisa 1.500 vítimas fatais, 89,3\% do total das admitidas no IML Central em 2015.

$\mathrm{Na}$ casuística, houve predomínio do sexo masculino $(75,7 \%)$ e raça branca $(59,6 \%)$. A idade média das vítimas foi de $49,7( \pm 23,6)$ anos. O tipo de trauma contuso prevaleceu $(75,7 \%)$ e a causa externa mais frequente foi quedas (33,5\%), seguida de agressões $(27,8 \%)$ e acidentes de transporte (22,5\%), que incluiu pedestres, ciclistas, motociclistas e ocupantes de automóvel (Tabela 1).

Tabela 1 - Características do evento traumático, região do corpo mais gravemente ferida e causa da morte das vítimas (n=1.500). São Paulo, Brasil, 2015.

\begin{tabular}{|c|c|c|}
\hline Variáveis & $\mathbf{N}$ & $\%$ \\
\hline \multicolumn{3}{|l|}{ Tipo de trauma } \\
\hline Contuso & 1.135 & 75,7 \\
\hline Penetrante & 365 & 24,3 \\
\hline \multicolumn{3}{|l|}{ Causa externa segundo CID-10* } \\
\hline Quedas (W01-W19) ${ }^{+}$ & 503 & 33,5 \\
\hline Agressões $(\mathrm{X} 85-\mathrm{Y} 09)^{\ddagger}$ & 418 & 27,8 \\
\hline \multicolumn{3}{|l|}{ Acidente de transporte $(\mathrm{V} 01-\mathrm{V} 69)^{\S}$} \\
\hline Pedestre/ciclista & 171 & 11,4 \\
\hline Motociclista & 100 & 6,7 \\
\hline Ocupante de automóvel & 66 & 4,4 \\
\hline Lesões autoprovocadas intencionalmente $(\mathrm{X} 70-\mathrm{X} 84)^{\|}$ & 157 & 10,5 \\
\hline Causa de origem desconhecida & 52 & 3,5 \\
\hline Outras causas (X00-X09, X36, W20, W54, W87) & 33 & 2,2 \\
\hline \multicolumn{3}{|l|}{ Região do corpo mais gravemente ferida } \\
\hline Cabeça/pescoço (sim) & 810 & 54,0 \\
\hline Face $(\operatorname{sim})$ & 6 & 0,4 \\
\hline Tórax (sim) & 418 & 27,9 \\
\hline Abdome/conteúdo pélvico (sim) & 152 & 10,1 \\
\hline Extremidades/cintura pélvica (sim) & 259 & 17,3 \\
\hline Superfície externa $(\operatorname{sim})$ & 12 & 0,8 \\
\hline \multicolumn{3}{|l|}{ Causa da morte } \\
\hline Traumatismo craniano e/ou cervical & 436 & 29,0 \\
\hline Complicações pós-trauma & 401 & 26,7 \\
\hline Hemorragia traumática grave & 283 & 18,9 \\
\hline Traumatismos múltiplos & 244 & 16,3 \\
\hline Outras causas ${ }^{* *}$ & 133 & 8,9 \\
\hline Causa indefinida & 3 & 0,2 \\
\hline
\end{tabular}

"CID-10: Classificação Estatística Internacional de Doenças e Problemas Relacionados à Saúde - 10ª Revisão; 'W01W19: Queda do mesmo nível por escorregão, tropeção, passos em falso, de patins ou empurrão ou queda de um 
Fatores associados ao tempo da morte de vítimas de trauma: estudo de coorte retrospectivo $\mid 8$

nível ao outro envolvendo cadeira, leito, mobília, equipamento de playground, escadas, andaime, de outras estruturas como laje, árvore, penhasco, mergulho em água ou outras sem especificação; 'X85-Y09: Agressão por meio de substâncias químicas, enforcamento, sufocamento e sufocação, disparo de arma de fogo, material explosivo, fumaça, fogo, chamas, gases, objeto cortante ou penetrante, objeto contundente, projeção de um lugar elevado ou permanência diante de um objeto em movimento, impacto de um veículo a motor, força física, negligência e abandono, maus tratos ou não especificada; ${ }^{\S}$ V01-V69: Pedestre, ciclista, motociclista, ocupante de um triciclo motorizado, ocupante de um automóvel, ocupante de uma caminhonete ou ocupante de um veículo de transporte pesado traumatizado; $\| X 70-X 84$ : Lesão autoprovocada intencionalmente por enforcamento, estrangulamento e sufocação, submersão, disparo de arma de fogo, explosivos, fumaça, fogo, chamas, fumaça, gases, objeto cortante ou penetrante, objeto contundente, precipitação de um lugar elevado ou permanência diante de um objeto em movimento, impacto de um veículo a motor ou meios não especificados; "X00 - X09: Exposição a fogo, fumaça, chamas e/ou combustão de substâncias inflamáveis; X36: Vítima de avalanche, desabamento de terra e outros movimentos da superfície terrestre; W20: Impacto causado por objeto lançado, projetado ou em queda; W54: Mordedura ou golpe provocado por cão; W87: Exposição à corrente elétrica não especificada; " Incluiu asfixia, trauma raquimedular, trauma no tórax/abdome, trauma de extremidades, queimaduras, eletrocussão e intoxicação por monóxido de carbono.

Cabeça/pescoço (54,0\%), tórax (27,9\%) e extremidades/cintura pélvica (17,3\%) foram as regiões do corpo mais gravemente feridas. As causas de morte com elevada frequência segundo os laudos das autópsias foram traumatismo craniano e/ou cervical $(29,0 \%)$ e complicações póstrauma (26,7\%) (Tabela 1). Em relação aos óbitos por complicações pós-trauma (n=401), destacaram-se as complicações infecciosas $(64,8 \%)$, pulmonares $(21,0 \%)$ e cardíacas $(10,7 \%)$.

Quanto à gravidade do trauma, os escores médios de ISS e NISS foram $21,6( \pm 15,3)$ e 27,7 $( \pm 17,4)$, respectivamente, sendo que ambos variaram de 1 a 75 pontos. As vítimas tiveram, em média, $2,4( \pm 1,3)$ regiões corpóreas afetadas e em 26 casos $(1,7 \%)$ todas as seis regiões descritas no ISS apresentavam algum tipo de lesão traumática.

O tempo entre a ocorrência da lesão e o óbito variou de dois minutos a 374 dias. A Figura 1 mostra que mais de 900 vítimas morreram nos primeiros cinco dias após a lesão (cada coluna da figura corresponde a cinco dias), com queda acentuada dos óbitos depois desse período. Um paciente permaneceu por quase um ano internado no hospital devido às sequelas do trauma e faleceu por infecção nosocomial. 
9 | Batista DVA, Cassiano C, Oberg LMC, Gianvecchio DM, Sousa RMC, Nogueira LS

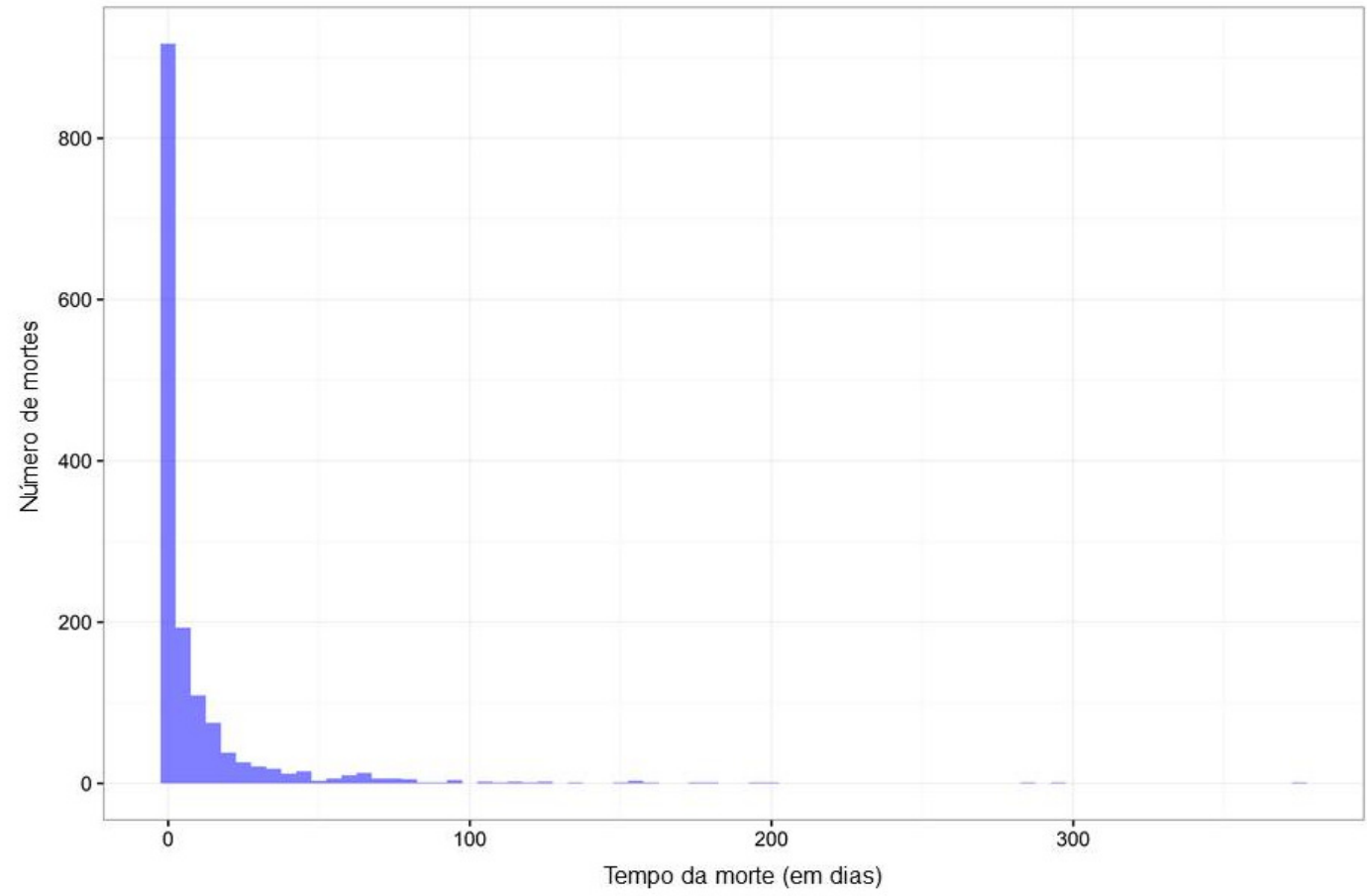

Figura 1 - Distribuição das vítimas de trauma $(n=1.500)$ segundo tempo da morte em dias. São Paulo, Brasil, 2015.

Um total de 830 vítimas morreu nas primeiras 24 horas após o trauma (Figura 2), sendo que, desses óbitos, 423 ocorreram na cena do evento.

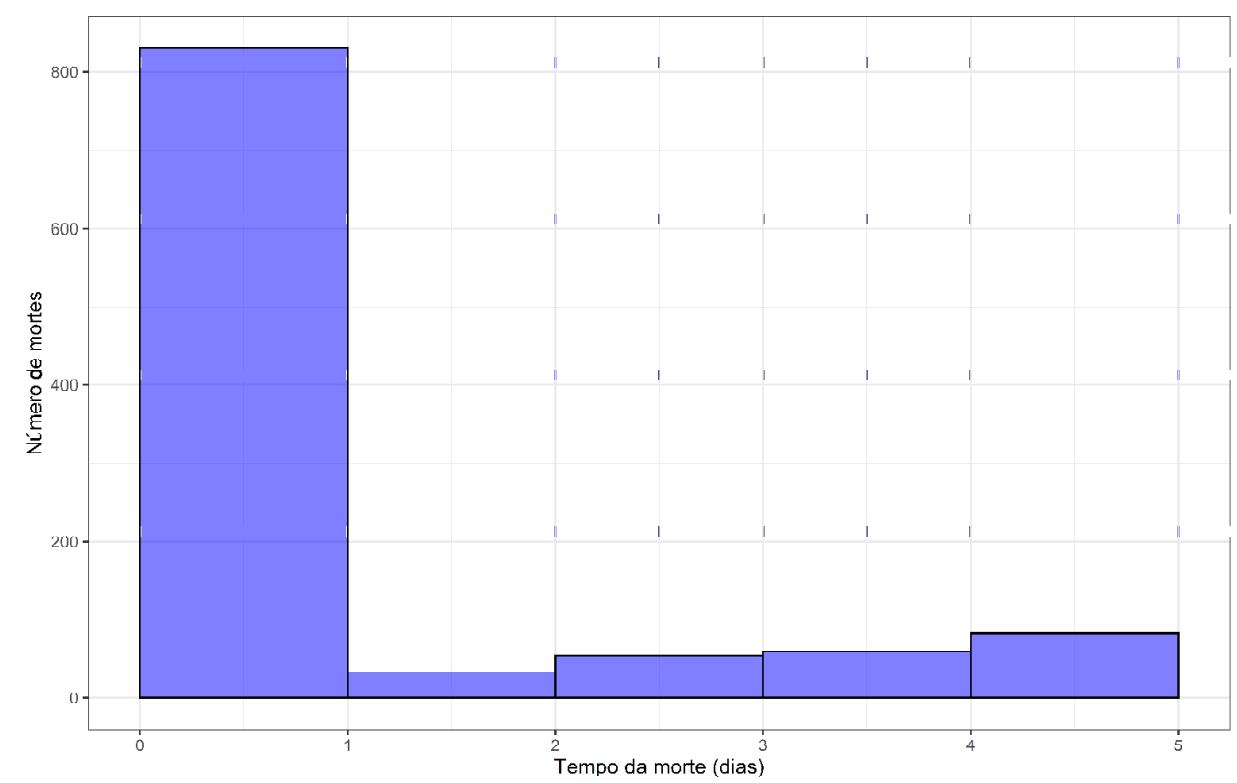

Figura 2 - Distribuição das vítimas de trauma (n=980) que evoluíram a óbito nos primeiros cinco dias após o trauma segundo tempo da morte. São Paulo, Brasil, 2015. 
Fatores associados ao tempo da morte de vítimas de trauma: estudo de coorte retrospectivo | 10

Os dados da Tabela 2 mostram que os fatores associados ao tempo de morte foram número de regiões corpóreas afetadas $(p<0,001)$, tipo de trauma $(p<0,001)$, sexo $(p=0,009), N I S S(p<0,001)$ e as causas externas agressões $(\mathrm{p}<0,001)$ e lesões autoprovocadas intencionalmente $(\mathrm{p}<0,001)$. Por outro lado, as variáveis idade, raça, ISS, região do corpo mais gravemente ferida e causa da morte não se associaram ao tempo de óbito das vítimas.

Tabela 2 - Modelo linear generalizado dos fatores associados ao tempo da morte das vítimas de trauma. São Paulo, Brasil, 2015.

\begin{tabular}{lcccc}
\multicolumn{1}{c}{ Variáveis } & Exp $\boldsymbol{\beta}$ & IC para Exp $\boldsymbol{\beta}$ (95\%) & $\mathbf{p}$ & VIF $^{*}$ \\
\hline $\begin{array}{l}\text { Número de regiões corpóreas afetadas } \\
\text { Tipo de trauma (ref contuso) }\end{array}$ & 0,50 & $0,47-0,54$ & $<\mathbf{0 , 0 0 1}$ & 1,09 \\
$\quad$ & & & 1,02 \\
$\quad$ Penetrante & 0,22 & $0,16-0,30$ & $<0,001$ & \\
Sexo (ref masculino) & & & & 1,17 \\
$\quad$ Feminino & 0,77 & $0,73-0,94$ & 0,009 & \\
NISS & 0,95 & $0,95-0,96$ & $<0,001$ & 1,22 \\
Causa externa (ref quedas) & & & & 1,15 \\
Pedestre/ciclista & 0,99 & $0,75-1,32$ & 0,975 & \\
Motociclista & 0,72 & $0,49-1,06$ & 0,099 & \\
Ocupante de automóvel & 1,19 & $0,79-1,82$ & 0,405 & \\
Agressões & 0,45 & $0,32-0,63$ & $<0,001$ & \\
Lesões autoprovocadas intencionalmente & 0,22 & $0,16-0,30$ & $<0,001$ & \\
Outras causas & 1,29 & $0,73-2,25$ & 0,387 & \\
Causa desconhecida & 0,81 & $0,51-1,26$ & 0,384 & \\
\hline
\end{tabular}

${ }^{*}$ Fator de Inflação de Variância; ${ }^{\dagger}$ Referência; ${ }^{*}$ New Injury Severity Score.

Ainda segundo dados da Tabela 2, é possível observar que a cada acréscimo de um ponto do NISS ou de uma região corpórea afetada reduziu em 5\% e 50\% o tempo de sobrevida dos traumatizados, respectivamente. As mulheres traumatizadas apresentaram menor tempo até o óbito (23\%) quando comparadas aos homens. Vítimas de agressão ou de lesões autoprovocadas intencionalmente morreram mais precocemente do que os doentes que sofreram queda, sendo que essa redução do tempo foi de $55 \%$ nas agressões e de $78 \%$ nas lesões autoprovocadas. As 
11 | Batista DVA, Cassiano C, Oberg LMC, Gianvecchio DM, Sousa RMC, Nogueira LS

vítimas de trauma penetrante apresentaram redução de $78 \%$ do tempo de sobrevida quando comparadas àquelas acometidas por algum tipo de lesão contusa.

A variação dos valores de VIF (de 1,02 a 1,22) mostrou que não houve colinearidade múltipla entre as variáveis que permaneceram no modelo final (Tabela 2). A pseudo $r^{2}$ foi de 0,81 , evidenciando capacidade preditiva moderada do modelo de regressão para fatores associados ao tempo da morte das vítimas de trauma.

\section{Discussão}

O estudo que descreveu o modelo de distribuição temporal de mortes por trauma foi o pioneiro ao explorar como as mortes ocorrem para criar estratégias de prevenção e implementação de melhorias na assistência, que resultaram em benefícios para os traumatizados após sua utilização. ${ }^{3}$ No entanto, as lesões por trauma afetam ainda milhares de pessoas em todo o mundo, com predomínio do sexo masculino e lesões contusas, ${ }^{5,20-23}$ assim como nesta investigação. Historicamente, os jovens têm sido as vítimas mais frequentes de trauma, porém, a epidemiologia das causas externas vem se modificando com o envelhecimento da população mundial, sendo a idade média próxima de 50 anos na atual investigação.

Entre as causas externas, as quedas $(33,5 \%)$ prevaleceram na amostra desta pesquisa. Esse resultado difere da maioria dos estudos que identificaram acidentes de trânsito ${ }^{4,9}$ ou ferimentos por arma de fogo ${ }^{24}$ como as principais causas externas de vítimas fatais. Entretanto, pesquisa publicada em 2017 comparou as mortes por trauma entre dois períodos temporais (2005-2006 e 2012-2013) e identificou que a principal causa de morte no primeiro período foi acidentes de trânsito, enquanto que no segundo prevaleceram as quedas, evidenciando o aumento desse tipo de causa externa entre as vítimas fatais. ${ }^{11}$ É importante destacar que, como resultado do envelhecimento, as quedas de idosos, especialmente no ambiente domiciliar, representam um 
Fatores associados ao tempo da morte de vítimas de trauma: estudo de coorte retrospectivo | 12

significativo problema de saúde pública, pois acarretam internação hospitalar prolongada e elevada taxa de mortalidade dessa população. ${ }^{25}$

A segunda causa externa mais frequente nesta pesquisa foi a agressão, que afetou $27,8 \%$ da amostra. Nesse contexto, ressalta-se a situação alarmante da violência urbana em São Paulo, local onde foi desenvolvida a presente pesquisa. Dados da Secretaria de Segurança Pública mostram que, em 2019, houve 2.906 vítimas por homicídio doloso e 3.364 tentativas de homicídio no Estado. ${ }^{26}$

Em relação à gravidade das vítimas desta pesquisa, a média do ISS foi de $21,6( \pm 15,3)$. O ISS também foi aplicado em outras investigações que analisaram vítimas fatais por trauma e evidenciaram escores médios superiores do índice: de 26,0 a $36,0 .{ }^{8,11}$ Com relação ao NISS $(27,7 \pm 17,4)$, identificou-se apenas um estudo ${ }^{27}$ que analisou a distribuição temporal dos óbitos e aplicou esse índice, com escore médio de 54,4 ( $\pm 19,9)$, resultado também acima do encontrado nesta pesquisa. Essa menor gravidade, em relação às demais investigações, pode estar associada ao elevado número de indivíduos, especialmente vítimas de queda, que morreram dias após a lesão e apresentavam baixos escores de ISS e NISS.

Cabeça/pescoço foi identificada como a região mais gravemente ferida em $54,0 \%$ da casuística e a conclusão da causa da morte descrita pelos médicos legistas confirmou o traumatismo craniano como a principal causa de morte das vítimas $(29,0 \%)$. Muitas pesquisas também descreveram o traumatismo cranioencefálico como mais frequente motivo de óbito entre as vítimas,,$^{5,8-9,11,23}$ reforçando que lesões graves nessa região do corpo são potencialmente letais. Vale ainda destacar que complicações pós-trauma e hemorragia traumática grave foram expressivas como causa da morte das vítimas deste estudo, assim como em outras investigações..$^{23,28}$

Nesta pesquisa, o tempo de sobrevida variou de poucos minutos a 374 dias, dados semelhantes aos resultados apresentados em uma investigação que analisou 260 laudos de 
13 | Batista DVA, Cassiano C, Oberg LMC, Gianvecchio DM, Sousa RMC, Nogueira LS

autópsias e identificou variação do intervalo lesão-morte de zero a 305 dias. ${ }^{27}$ Além disso, o maior percentual de mortes na casuística ocorreu nos primeiros cinco dias após a lesão, especialmente nas primeiras 24 horas, seguido de queda acentuada dos óbitos após esse período. Tal padrão de mortes ocorridas nas primeiras horas após o trauma com declínio ao longo do tempo também é evidenciado em outros estudos.,16

$\mathrm{Na}$ análise dos fatores associados à variável dependente deste estudo, verificou-se que as vítimas do sexo feminino apresentaram tempo de sobrevida $23 \%$ menor quando comparados aos homens, e que a cada acréscimo de um ponto do NISS ou de uma região corpórea afetada reduziu em $5 \%$ e $50 \%$ esse período, respectivamente. Quanto às causas externas, os principais destaques foram as vítimas de lesões autoprovocadas intencionalmente ou agressões, que morreram mais precocemente do que os doentes que sofreram queda. Além disso, foi possível observar que as vítimas de trauma penetrante tiveram tempo de sobrevida $78 \%$ menor quando comparadas àquelas que sofreram lesões contusas.

A diversidade das variáveis estudadas, assim como a ausência de investigações que analisaram o tempo entre a lesão e a morte como variável contínua, dificulta a comparação desses achados. No entanto, algumas análises podem ser confrontadas com estudos realizados nos Estados Unidos que investigaram os fatores de risco das mortes por trauma de acordo com a distribuição temporal. $., 7,24$

Na análise de 678 óbitos ocorridos em 2005, as lesões autoprovocadas intencionalmente (OR = 5,0; IC95\% = 2,7-9,5), a intoxicação alcoólica $(\mathrm{OR}=7,0 ; \mathrm{IC} 95 \%=2,8-7,2)$, a asfixia $(\mathrm{OR}=17,8$; IC95\% = 2,3-14,2) e as lesões na cabeça $(\mathrm{OR}=2,2 ; \mathrm{IC} 95 \%=1,2-4,2)$ e no tórax $(\mathrm{OR}=3,6 ; \mathrm{IC} 95 \%=$ 1,5-8,8) foram preditores independentes de mortes imediatas. ${ }^{24}$ Outro estudo identificou que, entre as vítimas que apresentavam trauma penetrante e escore AIS na região abdominal maior ou igual a 4, 83\% das mortes ocorreram nas primeiras 24 horas do trauma. ${ }^{7}$ A investigação de 44.911 vítimas fatais por trauma mostrou que as lesões no tórax $(\mathrm{OR}=2,03)$ e abdome/pelve $(\mathrm{OR}$ 
Fatores associados ao tempo da morte de vítimas de trauma: estudo de coorte retrospectivo | 14

$=1,39)$, além do mecanismo de trauma penetrante $(\mathrm{OR}=2,55)$, foram preditores de mortes precoces. ${ }^{6}$ Semelhante aos resultados encontrados na presente investigação, os estudos citados anteriormente evidenciaram que gravidade das lesões traumáticas (AIS $\geq 4),{ }^{7}$ trauma penetrante ${ }^{6-}$ ${ }^{7}$ e lesões autoprovocadas intencionalmente ${ }^{24}$ foram fatores de risco para mortes imediatas ou precoces, ou seja, menor tempo de sobrevida das vítimas.

Conforme identificado na regressão logística, o aumento do número de regiões corpóreas acometidas na vítima reduziu o tempo de sobrevida na amostra. Tal fato pode estar associado a maior probabilidade de, nesse contexto, ocorrer lesões em diferentes órgãos vitais e, consequentemente, falência múltipla de órgãos, condição que diminui significativamente a chance de sobrevida do traumatizado. ${ }^{29}$

O presente estudo mostrou que o pico das mortes nas primeiras horas pós-trauma se manteve, mesmo com os avanços e a melhora da qualidade da assistência nos contextos pré e intra-hospitalar. Nesse sentido, vale ressaltar que os traumas que envolveram a intencionalidade de provocar a morte (lesões autoprovocadas intencionalmente, agressões e ferimentos penetrantes) diminuíram o tempo de sobrevida das vítimas da pesquisa, enfatizando, assim, a necessidade de se aprimorar políticas de prevenção e de melhoria das condições de vida da população, com foco na redução das desigualdades sociais e garantia da assistência à saúde mental dos indivíduos.

Não menos importante, encontram-se as quedas, causa externa mais frequente neste estudo, cujas vítimas apresentavam maior tempo de sobrevida e baixa gravidade. Considerando que a elevação do número de quedas tem relação com o envelhecimento da população, em ascensão no Brasil, ${ }^{30}$ a prevenção desse evento com medidas simples e eficazes, especialmente no ambiente domiciliar, deve ser intensificada.

Além disso, os dados desta pesquisa revelaram elevada frequência de mortes por complicações pós-trauma, eventos considerados evitáveis e, muitas vezes, associados a erros 
15 | Batista DVA, Cassiano C, Oberg LMC, Gianvecchio DM, Sousa RMC, Nogueira LS

assistenciais. Nesse sentido, destaca-se a importância da capacitação e atualização periódica dos profissionais que prestam assistência aos traumatizados, assim como a garantia, pela instituição, de adequadas condições de trabalho.

Como limitação da presente investigação, deve-se considerar que a coleta de dados foi realizada em um IML com cobertura de algumas regiões específicas da cidade de São Paulo, o que pode trazer possíveis restrições à generalização dos resultados. Além disso, algumas variáveis que podem exercer influência no tempo da morte das vítimas não fizeram parte das análises deste estudo, visto que não são conteúdos dos laudos de autópsia, fonte primária da coleta dos dados desta pesquisa.

Para finalizar, é importante destacar que a escassez de investigações que retratam a variável tempo da morte resulta em dificuldade de identificar, com precisão, o tempo entre a ocorrência da lesão traumática e a confirmação do óbito, especialmente nas mortes imediatas. No entanto, a qualidade dos registros (laudos de autópsias e boletins de ocorrências) analisados nesta pesquisa permitiu a identificação precisa desse tempo e, complementarmente, a identificação de fatores associados.

\section{Conclusão}

Os resultados deste estudo evidenciaram, na análise de 1.500 laudos de autópsia, um importante pico de morte na primeira hora pós-trauma, com redução progressiva ao longo do tempo e permitiram identificar que os fatores associados ao tempo da morte das vítimas de trauma foram sexo, causas externas, lesões autoprovocadas intencionalmente e agressões, tipo de trauma, número de regiões corpóreas afetadas e gravidade do trauma. Portanto, conclui-se que mulheres, vítimas de traumas que envolvem a intencionalidade de provocar a morte e que apresentam elevada gravidade têm o tempo de sobrevida reduzido após a(s) lesão(ões). A preocupação com a qualidade da assistência às vítimas de trauma tem gerado muitas discussões em todo o mundo. Desse 
Fatores associados ao tempo da morte de vítimas de trauma: estudo de coorte retrospectivo | 16

modo, os resultados desta investigação trazem evidências importantes para a definição de políticas públicas de prevenção de eventos traumáticos e para a estruturação e organização dos serviços que atendem traumatizados com foco na melhoria da sobrevida dessa população.

Neste sentido, destaca-se a necessidade de incrementar a capacitação de todos os profissionais que prestam assistência aos traumatizados e aprimorar a articulação entre os serviços da rede de saúde, principalmente de urgência e emergência, com o objetivo de prestar um atendimento mais rápido, eficaz e integral às vítimas. Para a enfermagem, que tem papel de destaque na estruturação, organização e articulação dos atendimentos pré e intra-hospitalar, o conhecimento dos fatores associados ao tempo da morte das vítimas traz importantes contribuições que instrumentalizam essa equipe na identificação de indivíduos com risco de morte precoce e necessidade de tratamento definitivo em um centro especializado.

\section{Referências}

1. World Health Organization (WHO). WHO Mortality Database [Internet]. Geneva: WHO; 2020 [cited 2020 Jan 20]. Available from: https://www.who.int/data/data-collection-tools/who-mortality-database

2. Ministério da Saúde (BR), Departamento de Informática do Sistema Único de Saúde (Datasus). Estatísticas Vitais. Óbitos por ocorrência segundo capítulo CID-10 [Internet]. Brasília (DF): Datasus; 2019 [acesso em 2020 jan 20]. Disponível em: http://tabnet.datasus.gov.br/cgi/tabcgi.exe?sim/cnv/obt10br.def

3. Trunkey DD. Trauma. Accidental and intentional injuries account for more years of life lost in U.S. than cancer and heart disease. Among the prescribed remedies are improved preventive efforts, speedier surgery and further research. Sci Am. 1983;249(2):28-35.

4. Abbasi H, Bolandparvaz S, Yadollahi M, Anvar M, Farahgol Z. Time distribution of injury-related inhospital mortality in a trauma referral center in South of Iran (2010-2015). Medicine (Baltimore). 2017;96(21):e6871. doi: https://doi.org/10.1097/MD.0000000000006871

5. Pfeifer R, Schick S, Holzmann C, Graw M, Teuben M, Pape HC. Analysis of injury and mortality patterns in deceased patients with road traffic injuries: an autopsy study. World J Surg. 2017;41(12):31119. doi: https://doi.org/10.1007/s00268-017-4122-4

6. Valdez C, Sarani B, Young H, Amdur R, Dunne J, Chawla LS. Timing of death after traumatic injury - 
a contemporary assessment of the temporal distribution of death. J Surg Res. 2015;200(2):604-9. doi: https://doi.org/10.1016/j.jss.2015.08.031

7. Bardes JM, Inaba K, Schellenberg M, Grabo D, Strumwasser A, Matsushima K, et al. The contemporary timing of trauma deaths. J Trauma Acute Care Surg. 2018;84(6):893-9. doi: https://doi.org/10.1097/TA.0000000000001882

8. Rauf R, Von Matthey F, Croenlein M, Zyskowski M, Van Griensven M, Biberthaler P, et al. Changes in the temporal distribution of in-hospital mortality in severely injured patients-an analysis of the TraumaRegister DGU. PLoS One. 2019;14(2):e0212095. doi: https://doi.org/10.1371/journal.pone.0212095

9. Hwang K, Jung K, Kwon J, Moon J, Heo Y, Lee JCJ, et al. Distribution of trauma deaths in a province of Korea: is "trimodal" distribution relevant today? Yonsei Med J. 2020;61(3):229-34. doi: https://doi.org/10.3349/ymj.2020.61.3.229

10. Wang T, Wang Y, Xu T, Li L, Huo M, Li X, et al. Epidemiological and clinical characteristics of 3327 cases of traffic trauma deaths in Beijing from 2008 to 2017: a retrospective analysis. Medicine (Baltimore). 2020;99(1):e18567. doi: https://doi.org/10.1097/MD.0000000000018567

11. Oyeniyi BT, Fox EE, Scerbo M, Tomasek JS, Wade CE, Holcomb JB. Trends in 1029 trauma deaths at a level 1 Trauma Center: impact of a bleeding control bundle of care. Injury. 2017;48(1):5-12. doi: https://doi.org/10.1016/j.injury.2016.10.037

12. Negoi I, Paun S, Hostiuc S, Stoica B, Tanase I, Negoi RI, et al. Mortality after acute trauma: progressive decreasing rather than a trimodal distribution. J Acute Dis. 2015;4(3):205-9. doi: https://doi.org/10.1016/j.joad.2015.03.001

13. Abhilash KPP, Sivanandan A. Early management of trauma: the golden hour. Curr Med Issues. 2020;18(1):36-9. doi: https://doi.org/10.4103/cmi.cmi_61_19

14. Cabral ELS, Castro WRS, Florentino DRM, Viana DA, Costa Junior JF, Souza RP, et al. Response time in the emergency services. Systematic review. Acta Cir Bras. 2018;33(12):1110-21. doi: https://doi.org/10.1590/s0102-865020180120000009

15. Clark DE, Qian J, Sihler KC, Hallagan LD, Betensky RA. The distribution of survival times after injury. World J Surg. 2012;36(7):1562-70. doi: https://doi.org/10.1007/s00268-012-1549-5

16. Pfeifer R, Teuben M, Andruszkow H, Barkatali BM, Pape HC. Mortality patterns in patients with multiple trauma: a systematic review of autopsy studies. PLoS One. 2016;11(2):e0148844. doi: https://doi.org/10.1371/journal.pone.0148844

17. Association for the Advancement of Automotive Medicine (AAAM). The Abbreviated Injury Scale (AIS): 2008, update 2015. Des Plaines: AAAM; 2015. 
Fatores associados ao tempo da morte de vítimas de trauma: estudo de coorte retrospectivo | 18

18. World Health Organization (WHO). International Statistical Classification of Diseases and Related Health Problems - 10th Revision [Internet]. Geneva: WHO; 2010 [cited 2020 Jan 10]. Available from: https://www.who.int/classifications/classification-of-diseases

19. Osler T, Baker SP, Long W. A modification of the injury severity score that both improves accuracy and simplifies scoring. J Trauma Acute Care Surg. 1997;43(6):922-5. doi: https://doi.org/10.1097/00005373199712000-00009

20. Miller RT, Nazir N, McDonald T, Cannon CM. The modified rapid emergency medicine score: a novel trauma triage tool to predict in-hospital mortality. Injury. 2017;48(9):1870-77. doi: https://doi.org/10.1016/j.injury.2017.04.048

21. Kimura A, Tanaka N. Reverse shock index multiplied by Glasgow Coma Scale score (rSIG) is a simple measure with high discriminant ability for mortality risk in trauma patients: an analysis of the Japan Trauma Data Bank. Crit Care. 2018;22(1):87. doi: https://doi.org/10.1186/s13054-018-2014-0

22. Serviá L, Badia M, Montserrat N, Trujillano J. Gravedad em pacientes traumáticos ingressados em UCI. Modelos fisiológicos y anatómicos. Med Intensiva. 2019;43(1):26-34. doi: https://doi.org/10.1016/j.medin.2017.11.008

23. Callcut RA, Kornblith LZ, Conroy AS, Robles AJ, Meizoso JP, Namias N, et al. The why \& how our trauma patients die: a prospective Multicenter Western Trauma Association study. J Trauma Acute Care Surg. 2019;86(5):864-70. doi: https://doi.org/10.1097/TA.0000000000002205

24. Gunst M, Ghaemmaghami V, Gruszeki A, Urban J, Frankel H, Shafi S. Changing epidemiology of trauma deaths leads to a bimodal distribution. Proc (Bayl Univ Med Cent). 2010;23(4):349-54. doi: https://doi.org/10.1080/08998280.2010.11928649

25. Whiteman C, Davidov DM, Sikora R, Paulson D, Schaefer G. Major Trauma and the Elder West Virginian: a six-year review at a Level I Trauma Center. W V Med J [Internet]. 2016 [cited 2020 Feb 20];112(3):94-9. Available from: https://www.ncbi.nlm.nih.gov/pmc/articles/PMC4926309/pdf/nihms796357.pdf

26. Estado de São Paulo, Secretaria de Segurança Pública. Dados estatísticos do estado de São Paulo [Internet]. São Paulo (SP): Secretaria de Segurança Pública; 2020 [acesso em 2020 set 05]. Disponível em: https://www.ssp.sp.gov.br/estatistica/mapas.aspx

27. Soreide K, Krüger AJ, Vardal AL, Ellingsen CL, Soreide E, Lossius HM. Epidemiology and contemporary patterns of trauma deaths: changing place, similar place, older face. World J Surg. 2007;31(11):2092-103. doi: https://doi.org/10.1007/s00268-007-9226-9

28. Abe T, Komori A, Shiraishi A, Sugiyama T, Iriyama H, Kainoh T, et al. Trauma complications and inhospital mortality: failure-to-rescue. Crit Care. 2020;24:223. doi: https://doi.org/10.1186/s13054-020-02951-1 
29. Hutchings L, Watkinson P, Young JD, Willett K. Defining multiple organ failure after major trauma: a comparison of the Denver, Sequential Organ Failure Assessment and Marshall scoring systems. J Trauma Acute Care Surg. 2017;82(3):534-41. doi: https://doi.org/10.1097/TA.0000000000001328

30. Veras RP, Oliveira M. Envelhecer no Brasil: a construção de um modelo de cuidado. Ciênc Saúde Colet. 2018;23(6):1929-36. doi: https://doi.org/10.1590/1413-81232018236.04722018

Editor Científico: Tânia Solange Bosi de Souza Magnago

Editor Associado: Etiane de Oliveira Freitas

Fomento / Agradecimento: O presente trabalho foi realizado com apoio da Coordenação de Aperfeiçoamento de Pessoal de Nível Superior - Brasil (CAPES) - Código de Financiamento 001

\section{Autor correspondente}

Lilia de Souza Nogueira

E-mail: lilianogueira@usp.br

Endereço: Avenida Doutor Enéas de Carvalho Aguiar, 419. Cerqueira César, São Paulo, SP, Brasil. CEP: 05403-000

\section{Contribuições de Autoria}

\section{1 - Daniela Vieira de Andrade Batista}

Concepção e desenho do estudo/pesquisa; análise e interpretação dos dados; revisão final com participação crítica e intelectual no manuscrito.

\section{2 - Carolina Cassiano}

Análise e interpretação dos dados; revisão final com participação crítica e intelectual no manuscrito.

\section{3 - Luciana Maria Capurro de Queiroz Oberg}

Análise e interpretação dos dados; revisão final com participação crítica e intelectual no manuscrito.

\section{4 - Daniele Muñoz Gianvecchio}

Análise e interpretação dos dados; revisão final com participação crítica e intelectual no manuscrito.

\section{5 - Regina Marcia Cardoso de Sousa}

Análise e interpretação dos dados; revisão final com participação crítica e intelectual no manuscrito.

\section{6 - Lilia de Souza Nogueira}

Concepção e desenho do estudo/pesquisa; análise e interpretação dos dados; revisão final com participação crítica e intelectual no manuscrito.

\section{Como citar este artigo}

Batista DVA, Cassiano C, Oberg LMC, Gianvecchio DM, Sousa RMC, Nogueira LS. Fatores associados ao tempo da morte de vítimas de trauma: estudo de coorte retrospectivo. Rev. Enferm. UfSM. 2021 [Acesso em: Ano Mês Dia]; vol.11 e29: 1-19. DOI:https://doi.org/10.5902/2179769247475 